I NRA Prod. Anim., 1999, 12 (3), 183-194
R. BAUMONT, P. CHAMPCIAUX, J . AGABRIEL, J . ANDRIEU, J. AUFRÈRE, B. MICHALET-DOREAU, C. DEMARQUILLY

INRA Uni té de Recherches sur les Herbi vores, Thei $x$, 63122 Sai nt-Genès-Champanelle

baumont@dermont.inra.fr

\section{Une démarche intégrée pour prévoir la valeur des aliments pour les ruminants: PrévAlim pour INRAtion}

\begin{abstract}
Le module PrévAlim, proposé avec la nouvelle version du logiciel INRAtion, permet à partir du résultat de l'analyse d'un fourrage ou d'un aliment concentré d'en estimer la valeur alimentaire (valeur nutritive UF et PDI, et valeur d'encombrement UE ) pour les ruminants. II intègre de façon hiérarchisée l'ensemble des outils proposés par l'INRA pour prévoir la valeur des aliments.
\end{abstract}

Les systèmes d'alimentation proposés en 1988 par I'INRA (UF, PDI et UE), mis en œuvre à l'aide du logiciel INRAtion, permettent d'établir des rations ajustant au mieux les

\section{Résumé}

Cet article présente les principes retenus pour élaborer le module de prévision de la valeur d'un aliment à partir des résultats de son analyse. Le module PrévAlim, proposé avec la nouvelle version d'I NRAtion, permet de calculer à la fois la valeur nutritive (UF, PDI) et pour les fourrages la valeur d'encombrement (UE) à partir de la nature de l'aliment et de la mesure de certaines de ces caractéristiques. Pour la valeur nutritive, une démarche séquentielle a été adoptée, centrée sur l'estimation de la dMO et de la dégradabilité de l'azote (DT) ; le calcul des valeurs UF et PDI utilise ensuite la séquence des équations des systèmes INRA 1988. L'estimation de la dMO se fait soit préférentiellement à partir de la méthode de digestibilité enzymatique pepsine-cellulase ( $d C s$ ou dC 0 ), soit à partir de la composition chimique ou de l'âge de la plante. L'estimation de la DT et de la valeur PDI des concentrés est basée sur la méthode enzymatique avec une protéase (DE 1). La valeur d'encombrement des fourrages est calculée à partir de l'ingestibilité estimée directement à partir de la composition chimique ou de la $\mathrm{dMO}$; les équations par grandes classes d'aliments ont été déterminées sur la base des Tables INRA 1988. Ce module rassemble, coordonne et met à jour des outils déjà disponibles (Tables de prévision de 1981 et équations publiées depuis) et des outils créés spécialement (estimation des UE). Les valeurs calculées (UF, PDI et UE ) sont cohérentes entre elles ainsi qu'avec celles des Tables. L'approche hiérarchisée adoptée permet de calculer les valeurs même avec des informations manquantes et de retenir la méthode d'évaluation la plus pertinente en cas d'informations redondantes. apports alimentaires aux besoins des animaux et à leur capacité d'ingestion. La valeur alimentaire d'une ration dépend des valeurs des fourrages et des concentrés qui la composent et des interactions entre ces aliments (interactions énergétiques, substitution de l'ingestion de fourrage par le concentré). La valeur alimentaire d'un aliment intègre deux notions : la valeur nutritive, qui permet d'évaluer la contribution de cet aliment à la couverture des besoins nutritionnels de l'animal, et l'ingestibilité, qui permet d'évaluer la quantité de cet aliment que l'animal peut ingérer. La valeur nutritive d'un aliment recouvre sa valeur énergétique exprimée en UF, sa valeur azotée exprimée en PDI et en LysDi et MetDi, et sa teneur en minéraux. L'ingestibilité d'un fourrage est exprimée par sa valeur d'encombrement en UE. Les aliments concentrés n'ont pas de valeur d'encombrement propre. Leur valeur d'encombrement est fonction de celle des fourrages de la ration et du taux de substitution de l'aliment concentré aux fourrages.

Les valeurs des aliments peuvent être lues dans les Tables proposées par Andrieu et al dans le "Livre Rouge " (INRA 1988), mais une estimation plus précise de la valeur d'un fourrage ou d'un concentré peut être obtenue à partir d'une analyse de laboratoire. Les 
Critères nutritionnels dMO : di gestili té de la matiè reorganique

MOF : mati ère organi que fermentescible

DT : dégradabi li téthéori que des matières azotées dans le rumen

dr : di gesti bi litéréelle des proté nes dans l'intestin

Composition chi miquedes aliments

MS : mati ère sèche

$M O$ : matière organi que

MAT : Matières Azotées

Totales

MG : Matières grasses estimées par l'extrait éthéré

$\mathrm{MM}$ : matières mi nérales (cendres)

CB : Cellulose brute (méthode Weende)

ADF : Ligno-cellulose (Acid

Detergent Fi ber, méthode van Soest)

ADL : Lignine (Acid

Detergent Lignin, méthode van Soest)

Méthodes enzymati ques dCs et dCo : di gesti bi li té pepsinecellulase exprimée en MS (dCs) ou en $\mathrm{MO}(\mathrm{dCo})$

DE1 : dégradation de pro té nes par la protéase au temps $1 \mathrm{~h}$ méthodes d'estimation de la valeur des aliments, qui reposaient principalement sur la composition chimique des aliments (INRA 1981), ont largement évolué au cours des vingt dernières années. Une méthode enzymatique associant la pepsine et une cellulase permet de prévoir la digestibilité des fourrages classiques et des aliments concentrés (Aufrère et MichaletDoreau 1983 et 1988, Aufrère et Demarquilly 1989) ainsi que celle du maïs plante entière (Andrieu et Aufrère 1996). Une méthode enzymatique utilisant une protéase permet d'estimer la valeur azotée des aliments concentrés (Aufrère et al 1989).

Du fait de l'existence de différentes méthodes et des modifications apportées aux systèmes d'expression de la valeur des aliments (INRA 1988), la mise en œuvre des calculs nécessaires pour estimer les paramètres de la valeur alimentaire s'avère complexe. J usqu'à présent il n'existait pas de module informatisé pour intégrer l'ensemble des outils proposés par l'INRA et estimer la valeur des aliments. Cet outil, PrévAlim, est maintenant disponible avec la nouvelle version d'INRAtion (2.7). PrévAlim permet, à partir du résultat d'une analyse, de calculer la valeur nutritive d'un aliment exprimée en UF et PDI ainsi que la valeur d'encombrement pour les fourrages et d'utiliser ces valeurs dans INRAtion. Cet article présente les trois principes retenus pour élaborer PrévAlim ainsi que leur mise en œuvre. Ces principes sont :

- intégrer de façon hiérarchisée l'ensemble des outils disponibles pour prévoir la valeur des aliments ;

- proposer une estimation de la valeur d'encombrement des fourrages chaque fois que cela est possible ;

- assurer la cohérence entre les prévisions des trois composantes UF, PDI et UE.

\section{1 / Valeur nutritive des fourrages}

Les modes d'expression de la valeur nutritive des aliments dans le système des UF
(Vermorel et al 1987), et dans le système PDI (Vérité et al 1987) sont rappelés dans le tableau 1. PrévAlim estime la valeur énergétique et azotée des fourrages selon la démarche séquentielle centrée sur l'estimation de la digestibilité de la matière organique (dMO) et de la dégradabilité de l'azote (DT) qui a servi à l'élaboration des Tables (Andrieu et Demarquilly 1987; tableau 1). La valeur minérale ne peut être estimée que par l'analyse. PrévAlim utilise donc les valeurs en minéraux de l'aliment de référence sélectionné dans les Tables. Celles-ci peuvent être modifiées par l'utilisateur dans INRAtion.

\section{1 / Digestibilité de la matière organique et valeur énergétique}

Pour la valeur énergétique, la démarche consiste essentiellement à estimer la dMO, puis les UFL et UFV sont calculées de façon séquentielle à partir des estimations de l'énergie brute, de l'énergie digestible, de l'énergie métabolisable et enfin de l'énergie nette (figure 1). Cette méthode assure le maximum de cohérence avec les Tables INRA (Andrieu et al 1988) et en conséquence aucune équation directe de prévision des valeurs UF n'est utilisée. Trois groupes de méthodes d'estimation de la dMO ont été intégrés dans PrévAlim :

- à partir de la digestibilité pepsine-cellulase $(\mathrm{dCs})$, qui peut être réalisée sur les fourrages verts, les foins et le maïs plante entière à la mise en silo (la teneur en MAT est également nécessaire dans ce dernier cas), mais pas sur les échantillons ensilés. L'ensemble des équations utilisant la dCs a été récapitulé par Aufrère et Graviou (1996) ;

- à partir de la composition chimique (cendres ou matières minérales : MM, cellulose brute : CB, matières azotées totales : MAT) ; cette méthode s'applique à l'ensemble des fourrages classiques (équations de prévision par espèce végétale et cycle de végétation, INRA 1981) et au maïs (Andrieu et Aufrère 1996) ;

- à partir de l'âge du fourrage, uniquement pour les fourrages verts (INRA 1981).

Figure 1. Prévision de la valeur nutritive des fourrages.

Critères

mesurés

lus dans les Tables

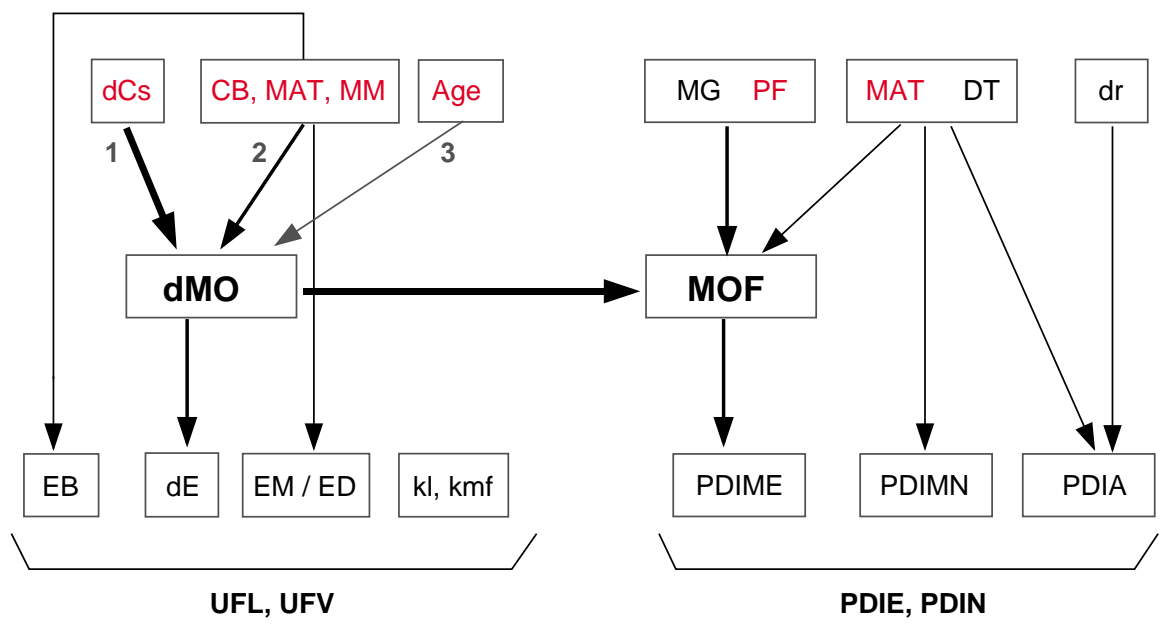


Tableau 1. Rappel des principes de calcul de la valeur des aliments.

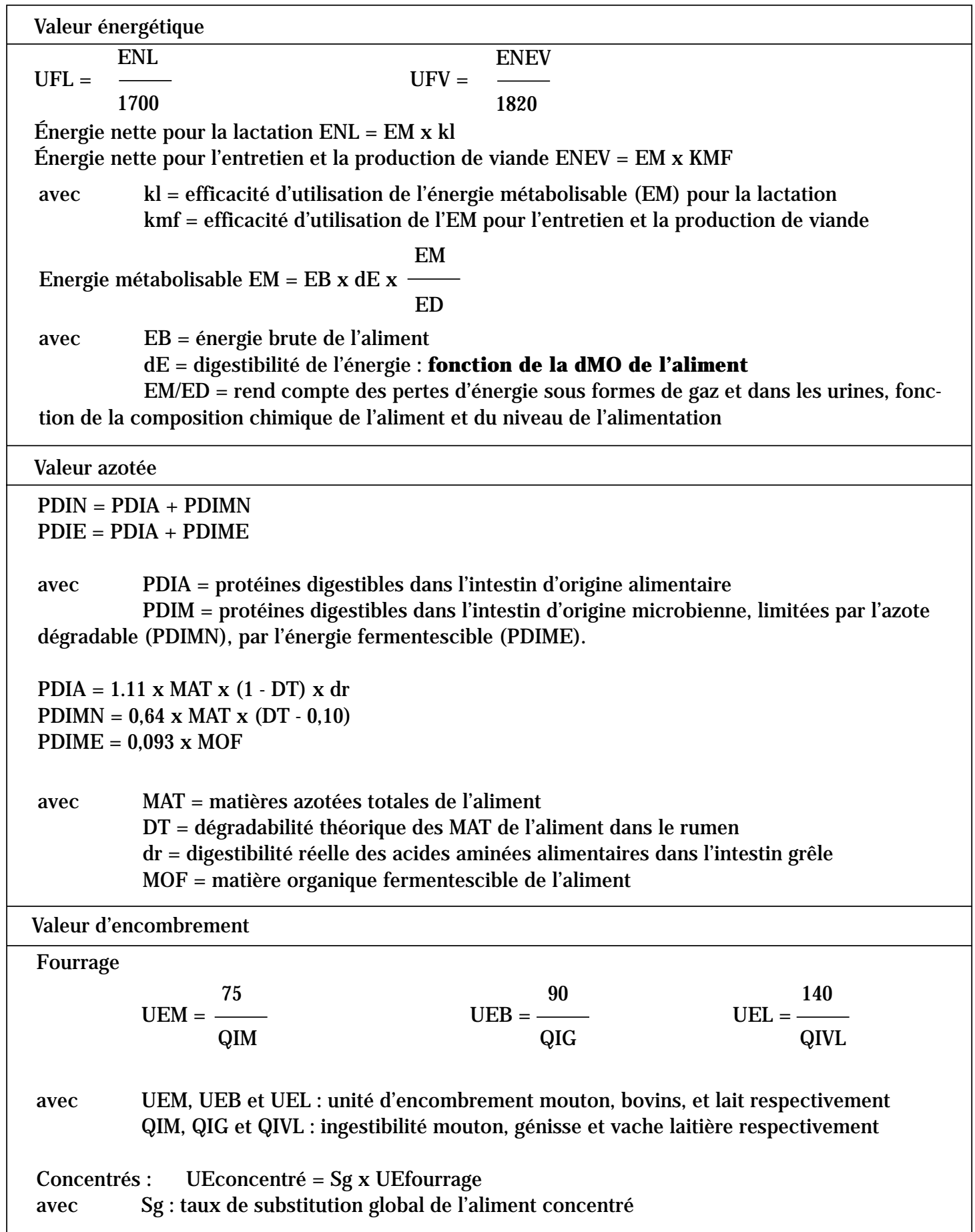

Un ordre de priorité dans l'utilisation de ces méthodes a été défini (figure 1). Le module utilise en priorité la prévision par la dCs, puis par la composition chimique, puis par l'âge. La dégradabilité à la pepsine-cellulase est une méthode plus générale que la composition chimique. Elle permet d'obtenir une prévision précise de la dMO, en particulier pour les associations de fourrages et les prairies naturelles. Les équations basées sur la composition chimique nécessitent une description plus détaillée du fourrage (espèce végétale, cycle de végétation) et sont moins précises lorsqu'elles sont utilisées pour des fourrages récoltés en fin de cycle. Dans ces cas là, leur utilisation peut entraîner des écarts par rapport aux valeurs des Tables. Lorsque les infor- mations saisies permettent de prévoir la dMO par deux méthodes différentes, le module les compare. Si l'écart entre les deux estimations de la dMO est supérieur à 3 points, un message d'alerte s'affiche et indique la méthode dont la valeur calculée se rapproche le plus de la dMO de la Table INRA. L'utilisateur reste bien entendu maître du choix de la méthode retenue et peut aller à l'encontre de la priorité proposée.

\section{2 / Valeur azotée}

Le calcul de la valeur azotée d'un aliment (PDI) nécessite de connaître, outre sa teneur en MAT et sa dMO, la dégradabilité théorique
La valeur nutritive des fourrages est calculée à partir des valeurs estimées de dMO et de DT des matières azotées, en utilisant les équations INRA 1988. 
de ses matières azotées dans le rumen (DT) et la digestibilité réelle des protéines dans l'intestin (dr). Les valeurs de DT et de dr (valeurs fixes par grands types d'aliment) ne peuvent pas actuellement être prévues pour les fourrages et sont donc celles indiquées dans les Tables (figure 1).

Pour l'estimation de la valeur PDIE, le module calcule la matière organique fermentescible (MOF) à partir de la dMO retenue par l'utilisateur pour prévoir la valeur énergétique. Ainsi la cohérence entre l'estimation des valeurs éner- gétique et azotée est assurée. Le calcul de la MOF utilise les valeurs de matières grasses (MG, estimées par l'extrait éthéré) des Tables. Les produits de fermentation des ensilages sont soit ceux indiqués dans les Tables, soit saisis par l'utilisateur si une analyse des produits de fermentation a été effectuée. Le module calcule également les teneurs en lysine digestible (LysDi) et en méthionine digestible (MetDi) à partir de paramètres spécifiques aux différentes catégories de fourrages et des valeurs PDIA et PDIE prévues (Rulquin et al 1998).

Tableau 2. Récapitulatif des principales équations de prévision utilisées dans PrévAlim.

\begin{tabular}{|c|c|c|}
\hline Variables & Caractéristiques de l'équation & Source \\
\hline \multicolumn{3}{|c|}{ dMO des prairies naturelles, graminées et légumineuse fourragères en vert et en foin } \\
\hline $\begin{array}{l}\mathrm{dCs} \\
\mathrm{dCs} \\
\text { CB, MAT } \\
\text { Age }\end{array}$ & $\begin{array}{l}\text { Equation générale prairie naturelle, } \\
\text { graminées et légumineuses en vert ou en foin } \\
\text { Trèfle blanc et mélanges à base de trèfle } \\
\text { Equations par espèce végétale et cycle } \\
\text { Equations par espèce végétale et cycle }\end{array}$ & $\begin{array}{l}\text { Aufrère et Graviou } 1996 \\
\text { Giovanni et al } 1992 \\
\text { INRA } 1981 \\
\text { INRA } 1981\end{array}$ \\
\hline \multicolumn{3}{|c|}{ dMO des prairies naturelles, graminées et légumineuses fourragères ensilées } \\
\hline \multicolumn{2}{|c|}{$\begin{array}{l}\text { Pas d'équations directes, on se ramène au fourrage vert } \\
\text { pour estimer la dMO de l'ensilage selon ses caractéristiques }\end{array}$} & INRA 1981 \\
\hline \multicolumn{3}{|c|}{ dMO du maïs plante entière et du maïs ensilage } \\
\hline $\begin{array}{l}\mathrm{dCs}, \mathrm{MAT} \\
\mathrm{CB}, \mathrm{MAT}\end{array}$ & $\begin{array}{l}\text { Equation plante entière extrapolée à l'ensilage } \\
\text { E quation plante entière extrapolée à l'ensilage }\end{array}$ & $\begin{array}{l}\text { Andrieu et Aufrère } 1996 \\
\text { Andrieu et Aufrère } 1996\end{array}$ \\
\hline \multicolumn{3}{|c|}{ dMO des aliments concentrés simples } \\
\hline $\begin{array}{l}\text { dCo } \\
\text { dCs ou dCo } \\
\text { CB ou ADF, } \\
\text { MAT }\end{array}$ & $\begin{array}{l}\text { Equation générale } \\
\text { Equation spécifique luzerne déshydratée } \\
\text { Céréales et sous-produits } \\
\text { Tourteaux de soja et de colza }\end{array}$ & $\begin{array}{l}\text { Aufrère et Michalet-Doreau } 1988 \\
\text { Aufrère et Graviou } 1996 \\
\text { Sauvant et al } 1987\end{array}$ \\
\hline \multicolumn{3}{|c|}{ dMO des aliments composés } \\
\hline $\begin{array}{l}\mathrm{dCo} \\
\mathrm{ADF}, \mathrm{ADL}\end{array}$ & & $\begin{array}{l}\text { Giger et al } 1990 \\
\text { Giger et al } 1990\end{array}$ \\
\hline \multicolumn{3}{|c|}{ DT et PDI des fourrages } \\
\hline \multicolumn{2}{|c|}{ Pas d'équation de prévision, calcul à partir des DT des Tables } & Vérité et al 1987 \\
\hline \multicolumn{3}{|c|}{ DT et PDI des aliments concentrés simples et composés } \\
\hline DE 1, MAT & & Aufrère et al 1989 \\
\hline \multicolumn{3}{|c|}{ Ingestibilité et valeur UE des prairies naturelles, graminées et légumineuses en vert, en foin et en ensilage } \\
\hline $\begin{array}{l}\text { dMO, MAT } \\
\text { CB, MAT } \\
\text { CB, MAT }\end{array}$ & $\begin{array}{l}\text { Equations ajustées sur les tables selon } \\
\text { famille, rspèce, cycle et mode de conservation } \\
\text { Equations ajustées sur les tables selon } \\
\text { famille, espèce, cycle et mode de conservation } \\
\text { Pour les prairies naturelles et les graminées en vert, } \\
\text { équations par espèce végétale et cycle }\end{array}$ & $\begin{array}{l}\text { PrévAlim } 1999 \\
\text { PrévAlim } 1999 \\
\text { INRA 1981, Dulphy \& al } 1987\end{array}$ \\
\hline \multicolumn{3}{|c|}{ Ingestibilité et valeurs UE de l'ensilage de maïs } \\
\hline dMO, MS & Equations ajustées sur les tables & PrévAlim 1999 \\
\hline
\end{tabular}


Tableau 3. Exemples d'utilisation de PrévAlim. Le module repose sur la base aliments d'INRAtion. La première opération consiste à choisir un aliment de référence dans la base, puis à saisir les valeurs issues de l'analyse de l'aliment.

Ensilage d'herbe : prévision à partir des caractéristiques du vert à la mise en silo

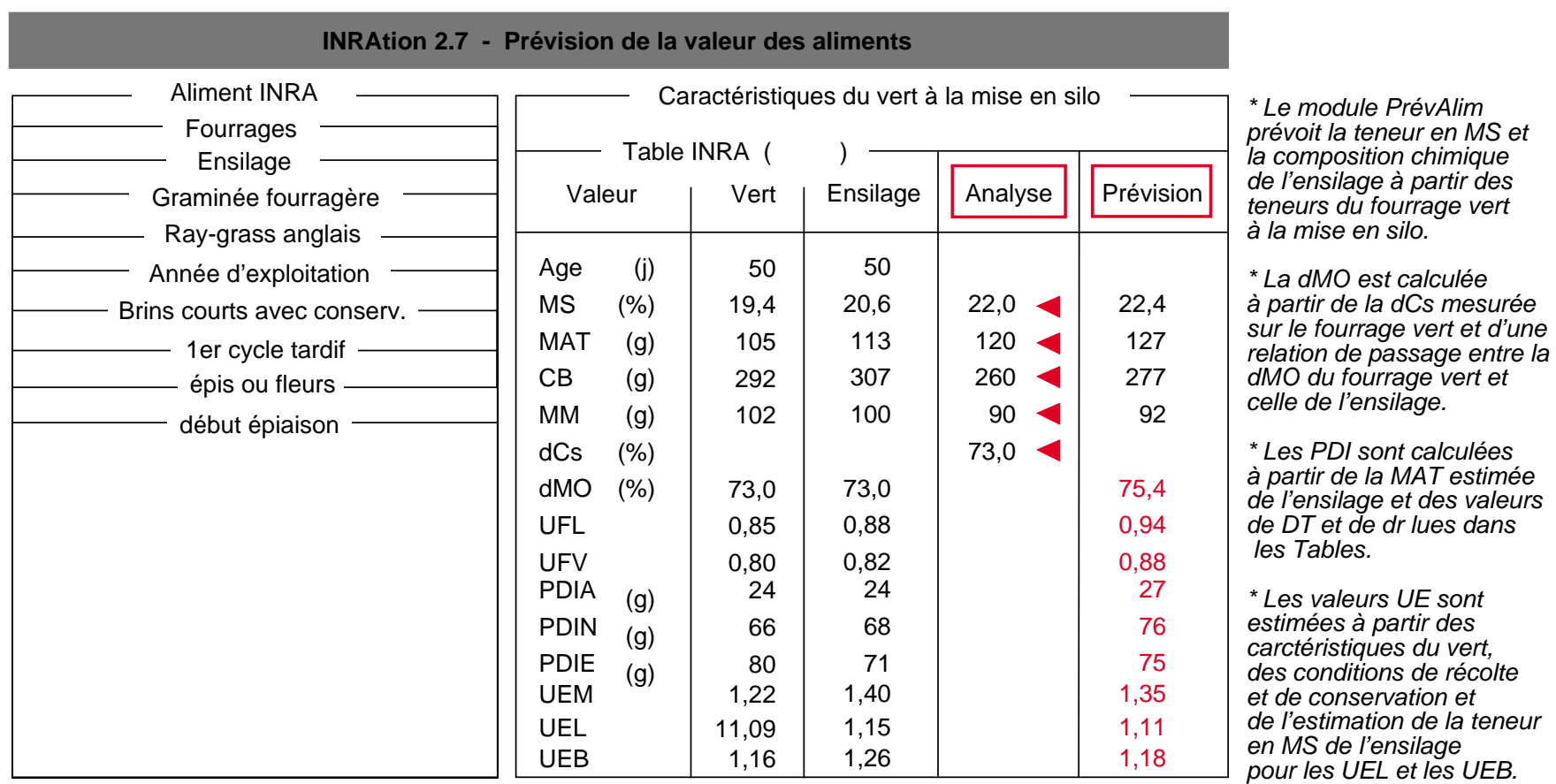

\section{Aliments composés}

* Il n'y a pas d'aliment de référence dans les Tables.

\section{INRAtion 2.7 - Prévision de la valeur des aliments}

Concentré composé

\begin{tabular}{|c|c|c|c|}
\hline \multicolumn{2}{|c|}{ Valeur / kg MS } & Analyse & Prévision \\
\hline MS & (\%) & $87,0<$ & \\
\hline MAT & (g) & $269<$ & \\
\hline ADF & (g) & $141<$ & \\
\hline ADL & (g) & $21<$ & \\
\hline MM & (g) & $87<$ & \\
\hline MG & (g) & $25<$ & \\
\hline DT & (\%) & & 71 \\
\hline $\mathrm{dr}$ & (\%) & & \\
\hline dCo & (\%) & $86,9<$ & \\
\hline DE1 & (\%) & $42,0<$ & \\
\hline $\begin{array}{l}\text { dMO } \\
\text { UFL }\end{array}$ & (\%) & & $\begin{array}{l}82,8 \\
0,97\end{array}$ \\
\hline $\begin{array}{l}\text { UFV } \\
\text { PDIA }\end{array}$ & (g) & & $\begin{array}{r}0,95 \\
74\end{array}$ \\
\hline PDIN & (g) & & 180 \\
\hline
\end{tabular}

\section{3 / Application aux différents types de fourrages}

Le tableau 2 récapitule l'origine des équations utilisées pour estimer la dMO.

Pour les fourrages verts les trois méthodes de prévision présentées dans la figure 1 peuvent être utilisées.

Pour les ensilages d'herbe le module affiche les valeurs de l'ensilage de référence choisi par l'utilisateur et celles du fourrage vert correspondant (voir exemple présenté dans le tableau 3). Deux voies pour la prévision de la valeur nutritive sont proposées, soit à partir d'un échantillon prélevé en vert à la mise en silo, soit à partir d'un échantillon d'ensilage; cependant, dans les deux cas, le calcul se fait toujours à partir de la composition et de la dMO du vert (mesurée ou estimée). Si l'analyse est faite sur un échantillon de fourrage vert prélevé à la mise en silo, la teneur en MS, la composition chimique de l'ensilage et la dMO de l'ensilage sont prévues 
à partir de celle du fourrage vert. Les trois méthodes de prévision de la dMO du fourrage à la mise en silo ( $\mathrm{dCs}$, composition chimique et âge) peuvent donc être utilisées. Si l'analyse est faite sur un échantillon d'ensilage, le module estime à partir de cette analyse la composition chimique et la dMO du fourrage vert à la mise en silo pour pouvoir ensuite estimer la dMO de l'ensilage. Seules les méthodes basées sur la composition chimique ou sur l'âge du fourrage à la coupe sont alors utilisables. En effet, il n'existe pas encore de relation disponible entre la dCs du fourrage vert et celle de l'ensilage. Si l'analyse des produits de fermentation est disponible, elle peut être saisie pour le calcul des produits de fermentation et du coefficient de correction de la MS de l'ensilage lié aux pertes de produits volatils lors du séchage à l'étuve. En l'absence de cette analyse, les valeurs sont celles des Tables.

Pour les foins, la prévision de la dMO peut être réalisée à partir de la dCs ou de la composition chimique du foin. Depuis quelques années on assiste à un fort développement de la récolte de fourrages enrubannés à des taux de MS qui peuvent varier entre 40 et $65 \%$. Les études réalisées sur ces fourrages (Andrieu et al 1992, Demarquilly et al 1998) ne sont pas encore assez complètes pour que les valeurs de ces fourrages soient intégrees dans les Tables. Pour estimer leur valeur, il est donc recommandé de choisir comme fourrage de référence un ensilage préfané lorsque le taux de MS du fourrage enrubanné est inférieur à $50 \%$ et un foin fané au sol par beau temps lorsque ce taux de MS est supérieur.

Pour les ensilages de maïs, la prévision de la dMO de la plante à la mise en silo peut être

Figure 2. Relation entre la dMO et l'ingestibilité des fourrages.

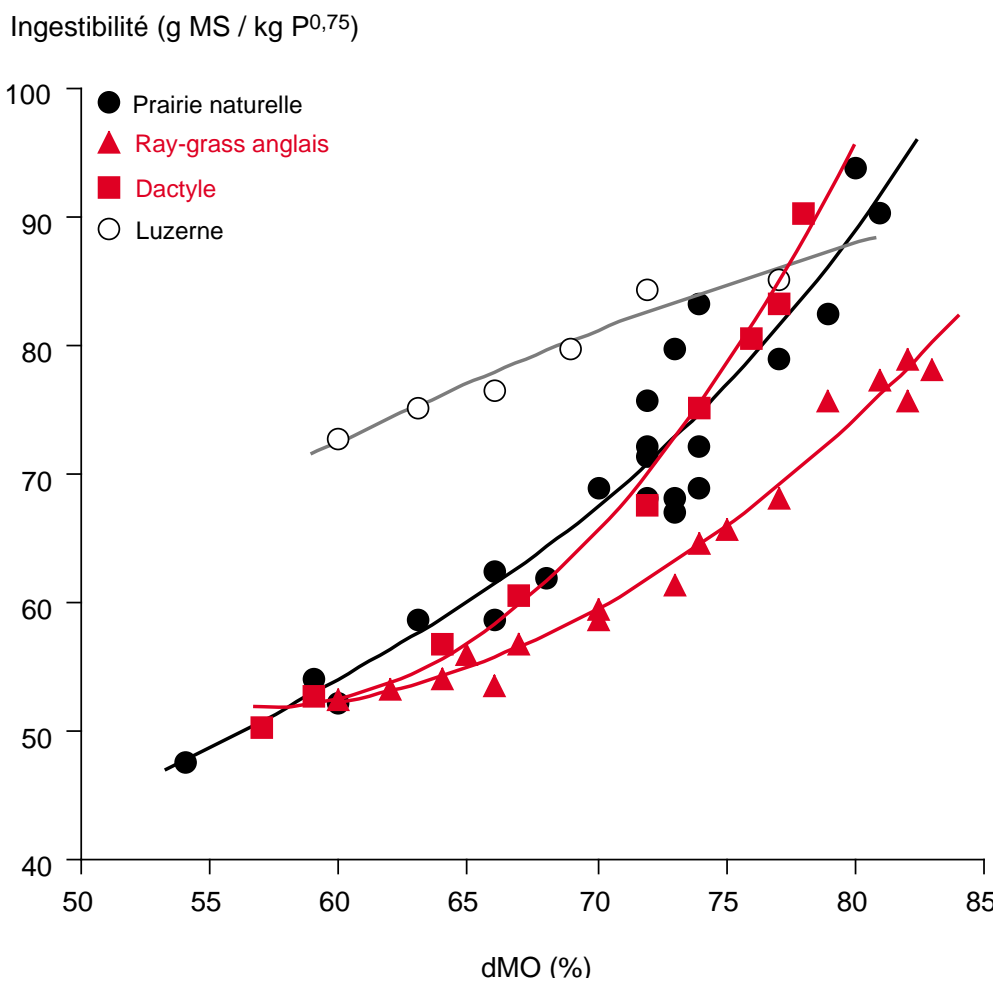

obtenue soit à partir des teneurs en MM, MAT et $C B$, soit à partir de la $\mathrm{dCs}$ et des teneurs en MM et MAT (Andrieu et Aufrère 1996). La digestibilité de la plante en vert est alors directement extrapolée à celle de l'ensilage si sa teneur en MS est supérieure à $25 \%$ (Andrieu 1985). La valeur énergétique de l'ensilage est calculée à partir de cette dMO et de la composition chimique de l'ensilage estimée à partir de celle de la plante en vert. L'équation utilisant la dCs est un peu plus précise que celle faisant appel à la composition chimique. C'est celle qui a été retenue pour l'inscription au catalogue de variétés de maïs par le groupe d'étude et de contrôle des variétés et des semences (GEVES). Si I'analyse est réalisée sur un échantillon d'ensilage, c'est une démarche identique à celle des ensilages d'herbe qui est proposée. Seule une prévision à partir de la composition chimique de l'ensilage est actuellement disponible.

L'utilisation d'associations fourragères se développe, que ce soit pour le pâturage (ray-grass, trèfle blanc) ou la récolte de fourrages conservés. A l'idéal, si des échantillons des fourrages constituant le mélange peuvent être analysés séparément, la valeur alimentaire du mélange peut alors être calculée à partir des valeurs des différents constituants. Mais, le plus souvent, seul le pourcentage des différents constituants du mélange pourra être apprécié. Le module permet d'estimer la valeur nutritive d'un mélange de deux fourrages de la façon suivante. Une valeur "Table" du mélange est d'abord calculée à partir des valeurs des deux constituants et de leurs pourcentages respectifs. Cette valeur "Table" représente la valeur de référence utilisée dans la partie prévision. L'estimation de la dMO du mélange ne peut être obtenue qu'à partir d'une mesure de la dCs du mélange. Pour les mélanges contenant du trèfle blanc, l'équation utilisée est celle publiée par Giovanni et al (1992). Les valeurs UF et PDI sont alors calculées à partir de la dMO prévue et des teneurs en MAT et en CB du mélange. Le module ne propose pas encore de prévision des valeurs UE pour les mélanges.

\section{2 / Ingestibilité et valeur d'encombrement des fourrages}

PrévAlim propose une approche rénovée qui permet d'estimer la valeur d'encombrement pour tous les fourrages pour lesquels l'estimation de la valeur nutritive est possible. La valeur d'encombrement d'un fourrage est une fonction inverse de son ingestibilité. Trois unités d'encombrement ont été définies (Dulphy et al 1987) respectivement pour les moutons (UEM), pour les vaches laitières (UEL) et pour les autres bovins (UEB) et dont les modes d'expression sont rappelés dans le tableau 1. Au cours d'un cycle de végétation, l'ingestibilité d'une espèce végétale donnée dépend comme la digestibilité, du stade de végétation. II existe par conséquent une liaison entre l'ingestibilité et la digestibilité (figure 2). La prévision de ces deux composantes de la valeur alimentaire doit donc être cohérente. 
Figure 3. Relation entre les valeurs d'ingestibilité (vaches laitières) lues dans les Tables et les valeurs calculées par les modèles de prévision retenus (cas des ensilages de prairie naturelle).

Ingestibilité calculée ( $\mathrm{g} \mathrm{MS} / \mathrm{kg} \mathrm{P}^{0,75}$ )

par le modèle MAT, CB et facteurs fixes

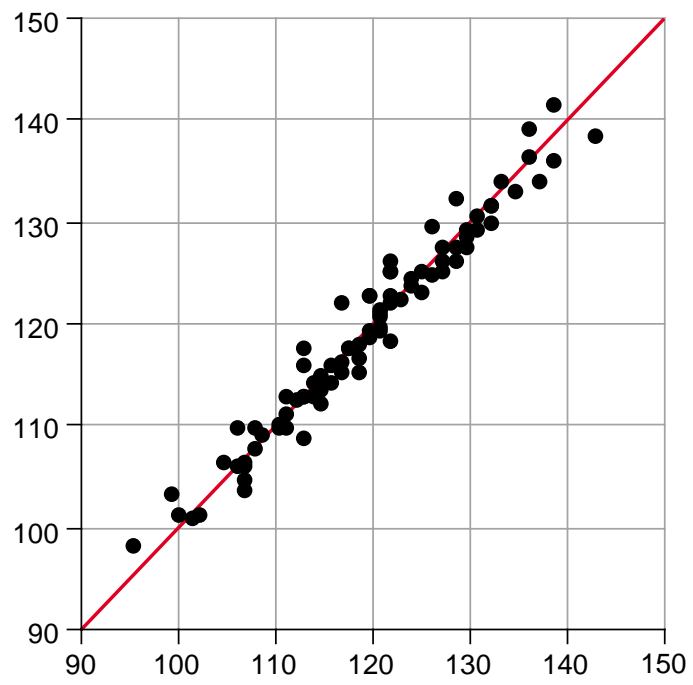

Ingestibilité calculée ( $\mathrm{g} \mathrm{MS} / \mathrm{kg} \mathrm{P}^{0,75}$ )

par le modèle dMO, MAT et facteurs fixes

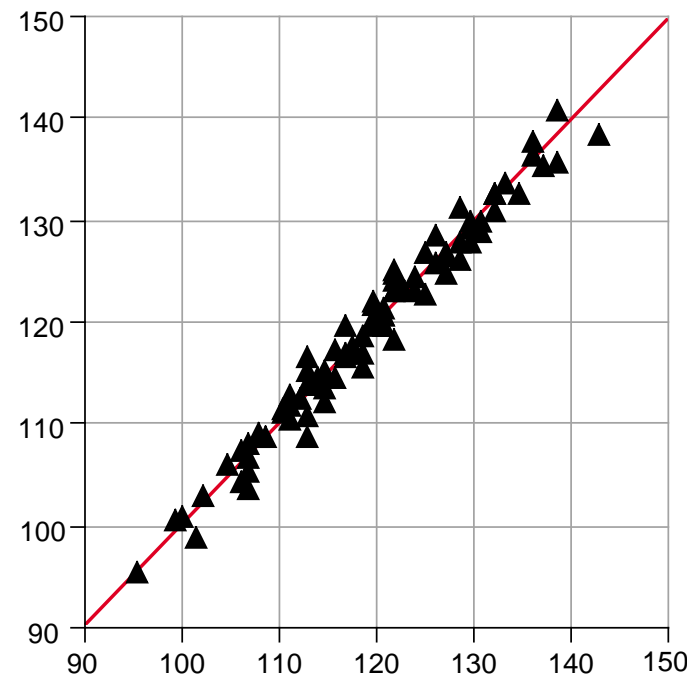

Ingestibilité figurant dans les Tables ( $\mathrm{g} \mathrm{MS} / \mathrm{kg} \mathrm{P}^{0,75}$ )

\section{$1,40 \longrightarrow$ UEL $\longrightarrow 1,00$ \\ 2.1 / Détermination et utilisation des valeurs d'encombrement}

$1,40 \longrightarrow$ UEL $\longrightarrow 1,00$

Valeurs d'encombrement correspondantes

Pour les fourrages verts, les valeurs UE des Tables (Andrieu et al 1988) ont été construites à partir des ingestibilités mesurées chez le mouton et des relations établies entre l'ingestibilité des fourrages verts chez le mouton et chez les bovins (génisses pour les UE B et vaches laitières pour les UEL, Dulphy et al 1987). Pour les foins et les ensilages, les valeurs mentionnées dans les Tables ont été calculées à partir de celles des fourrages verts correspondants et des relations rendant compte des modifications d'ingestibilité entraînées par la conservation (Andrieu et Demarquilly 1987). L'ingestibilité de certains fourrages par les bovins est le résultat de mesures réalisées directement sur génisses et vaches laitières. C'est le cas des foins et des ensilages de prairie naturelle de demi-montagne et de luzerne ainsi que de l'ensilage de maïs.

Les valeurs d'encombrement permettent de prévoir les quantités ingérées de fourrage et les taux de substitution entre les fourrages et les aliments concentrés. Elles sont donc une composante essentielle des calculs de ration effectués par INRAtion. Pour une vache produisant $30 \mathrm{~kg}$ de lait, une variation de $10 \%$ de la valeur UEL autour d'une valeur moyenne de 1 UEL entraîne une variation de l'apport recommandé de concentré de plus de $2 \mathrm{~kg}$ de matière sèche. Or actuellement peu de laboratoires estiment les valeurs UE à partir des analyses de fourrage et le rationnement fait le plus souvent appel aux valeurs UE des Tables. En effet, la valeur d'encombrement des fourrages (UE) peut être prévue pour les moutons (UEM) à partir de la composition chimique (INRA 1981), mais, jusqu'à présent, les valeurs d'encombrement pour les bovins (UEB et UEL) pouvaient difficilement être estimées autrement que par lecture des Tables (Andrieu et Demarquilly 1987). La révision des valeurs UE a permis d'intégrer l'ensemble des connaissances acquises sur les facteurs de variation de l'ingestibilité, mais a entraîné des limites pour la prévision des valeurs UE. Ainsi l'estimation de valeurs UE pour les bovins à partir d'analyses faites sur le fourrage conservé (foin ou ensilage) était jusqu'à présent impossible en pratique.

\section{2 / Méthode retenue pour esti- mer les valeurs d'encombrement}

PrévAlim propose une méthode d'estimation des valeurs UE basée sur les valeurs d'ingestibilité présentes dans les Tables de Andrieu et al (1988). La méthode retenue intègre tous les facteurs de variation de l'ingestibilité des fourrages pris en compte dans ces Tables et assure la cohérence des estimations des valeurs UE et de la valeur énergétique.

La cohérence avec les Tables INRA a été obtenue par le calcul d'équations reliant directement les ingestibilités chez les moutons, les génisses et les vaches laitières (QIM, QIG et QIVL) données dans les Tables et les valeurs de dMO, MAT et CB qui correspondent. Les équations ont été calculées en distinguant trois groupes de fourrages (prairies permanentes, graminées fourrageres et légumineuses fourragères) et trois modes d'exploitation (vert, foin et ensilage). Pour chacun des neuf cas définis, deux types de relations ont été calculés, reliant les ingestibilités soit à la dMO, aux MAT et à des facteurs fixes,
Les valeurs d'encombrement des fourrages sont calculées par un modèle utilisant soit dMO et MAT, soit MAT et CB si la dMO a été estimée par ces critères. 
Figure 4. Prévision de la valeur d'encombrement des fourrages.

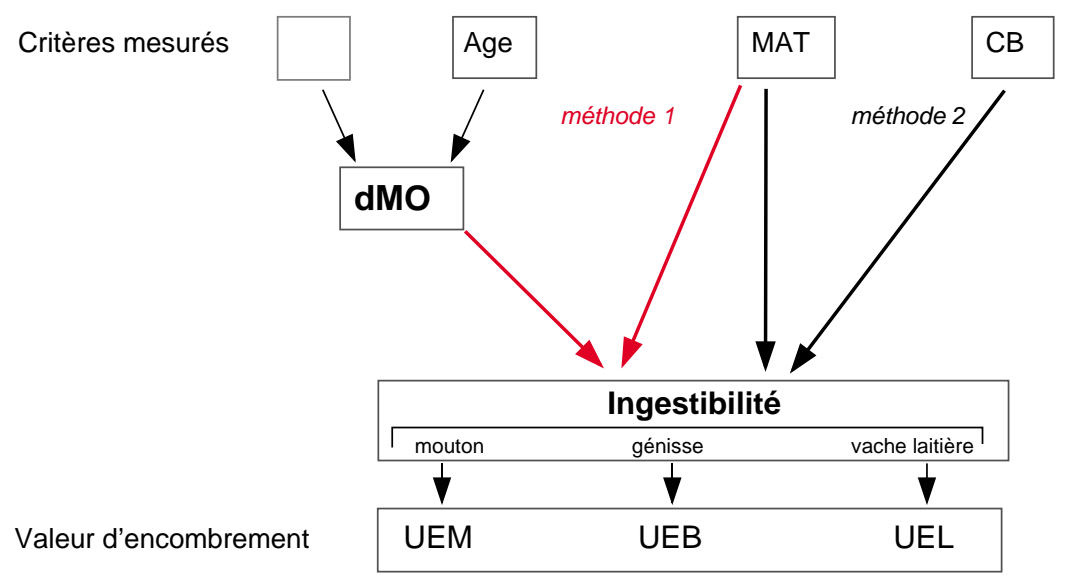

soit aux MAT, à la $C B$ et à des facteurs fixes. Les facteurs fixes utilisés dans ces équations sont ceux répertoriés dans les Tables (espèce végétale ou type pour les prairies permanentes, cycles de végétation, conditions de récolte et de conservation pour les foins et les ensilages). Ces équations expliquent entre 86 et $91 \%$ de la variabilité de l'ingestibilité des foins présents dans les Tables et entre 91 et $97 \%$ de celle des ensilages. Les équations basées sur la dMO et la teneur en MAT ont une précision comparable à celle des équations basées sur les teneurs en CB et en MAT (figure 3).

Afin d'assurer la cohérence entre la prévision de la dMO et l'estimation des valeurs UE, celle-ci est réalisée à partir des équations utilisant la dMO et la teneur en MAT lorsque la dMO est prévue à partir de la digestibilité pepsine cellulase, de l'âge ou saisie directement (figure 4 méthode 1) ou bien à partir des équations utilisant les teneurs en $C B$ et en MAT lorsque la dMO est prévue à partir de ces critères (figure 4 méthode 2 )

\section{3 / Application aux différents types de fourrages}

L'origine des équations utilisées pour estimer les valeurs UE est récapitulée dans le tableau 2.

Pour les fourrages verts de prairies permanentes et de graminées fourragères les équations de prévision de l'ingestibilité chez le mouton (INRA 1981) et les relations de passage aux ingestibilités chez les bovins (Dulphy et al 1987) continuent d'être appliquées dans le cas d'une prévision à partir de MAT et CB.

Dans le cas des ensilages d'herbe l'estimation de l'ingestibilité est proposée soit à partir des valeurs ( $d M O$, MAT et $C B$ ) du fourrage vert correspondant (prévision à la mise en silo), soit à partir des valeurs d'un échantillon d'ensilage. Pour les ensilages directs, la prévision des valeurs UEB et UEL intègre l'augmentation d'ingestibilité de l'ensilage avec sa teneur en MS entre 15 et $25 \%$, selon la méthode proposée par Demarquilly et Andrieu (1988).

Pour les foins, seule la prévision à partir d'une analyse du fourrage sec est proposée.
Pour les fourrages enrubannés, comme pour la prévision de la valeur nutritive, il est recommandé de choisir comme aliment de référence un ensilage préfané lorsque le taux de MS est inférieur à $50 \%$ et un foin fané au sol par beau temps lorsque taux de MS est supérieur à $50 \%$. En effet l'ingestibilité de ces fourrages enrubannés est proche de celle des foins récoltés au même stade de végétation dans de bonnes conditions (Andrieu et al 1992, Baumont et al 1997, Demarquilly et al 1998).

Le calcul des valeurs UE de l'ensilage de maïs a été traité à part. Les principaux facteurs qui rendent compte de la variabilité de son ingestibilité dans les Tables sont la dMO et la teneur en MS. Ainsi les valeurs UEM estimées dépendent de la dMO et de la dMO au carré, et les valeurs UEB et UEL dépendent de la dMO et de la teneur en MS. L'équation utilisée pour estimer les UEM explique $82 \%$ de la variabilité présente dans les Tables et celles utilisées pour les UEB et les UEL expliquent respectivement 98 et $97 \%$ de la variabilité. L'utilisation des valeurs UE prévues pour l'ensilage de maïs est en adéquation satisfaisante avec les quantités ingérées mesurées chez des vaches de réforme de race à viande $\left(r^{2}=0,85, n=16\right.$, synthèse des données obtenues par Agabriel et al). Pour les vaches laitières en production, les valeurs UEL prévues estiment de façon satisfaisante les quantités ingérées d'ensilage mesurées dans l'étude de Valentin et al (1999). En revanche, les valeurs prévues sous-estiment d'environ $10 \%$ les quantités mesurées dans les études de Andrieu et al (1997) et de Dubin et al (1998), mais cette sous-estimation est moindre que lorsqu'on utilise les valeurs des Tables. La sous-estimation des quantités ingérées d'ensilage dans certaines situations pourrait en partie s'expliquer par le taux élevé de couverture des besoins en PDI pratiqué pour les vaches laitières à haut niveau de production, qui a pour effet d'augmenter leur niveau d'ingestion (Faverdin et al 1998).

\section{3 / Prévision de la valeur nutritive des concentrés}

Pour les aliments concentrés, deux cas doivent être distingués : les matières premières ou aliments concentrés simples qui sont répertoriés dans les Tables du moins pour les plus couramment utilisées, et les concentrés composés qui sont un mélange de différents ingrédients. Comme pour les fourrages, la démarche retenue pour calculer la valeur nutritive des aliments concentrés consiste à estimer la dMO puis à calculer la valeur UF par la démarche séquentielle. Le calcul des valeurs PDI utilise la dMO estimée pour calculer la MOF. A la différence des fourrages, la DT des aliments concentrés (ou directement les valeurs PDI dans le cas des aliments composés) peut être estimée par une méthode enzymatique (DE 1, Aufrère et al 1989). 


\section{1 / Digestibilité de la matière organique et valeur énergétique}

Pour les aliments concentrés simples (figure 5, tableau 2), le module permet de prévoir la dMO à partir de la digestibilité pepsinecellulase exprimée en matière organique $(\mathrm{dCo})$, selon l'équation proposée par Aufrere et Michalet-Doreau (1988). Pour certains groupes de matières premières (blé, orge et maïs ainsi que leurs sous-produits respectifs ; tourteaux de soja et de colza), la dMO peut aussi être estimée à partir de la teneur en CB ou en ADF ou encore à partir de la teneur en MAT pour les tourteaux de soja et de colza (Sauvant et al 1987). Les différentes étapes du calcul des valeurs UFL et UFV des aliments concentrés simples sont effectuées avec les la deuxième utilise les teneurs en ADF et en ADL de l'aliment (figure 6, tableau 2). Le calcul des valeurs UFL et UFV à partir de la dMO et de la composition chimique de l'aliment utilise les équations spécifiques proposées pour les aliments composés (Giger et al 1990).

L'ordre de priorité d'utilisation des équations d'estimation de la dMO des aliments concentrés est le même que pour les fourrages. Le module utilise en priorité les équations basées sur la dégradabilité pepsine-cellulase (dCo ou dCs) par rapport aux équations basées sur la composition chimique. Comme pour les fourrages, l'utilisateur reste maître du choix de l'équation utilisée et peut vérifier la cohérence des estimations avec les valeurs des Tables pour les aliments simples.

Figure 5. Prévision de la valeur nutritive des aliments concentrés simples.

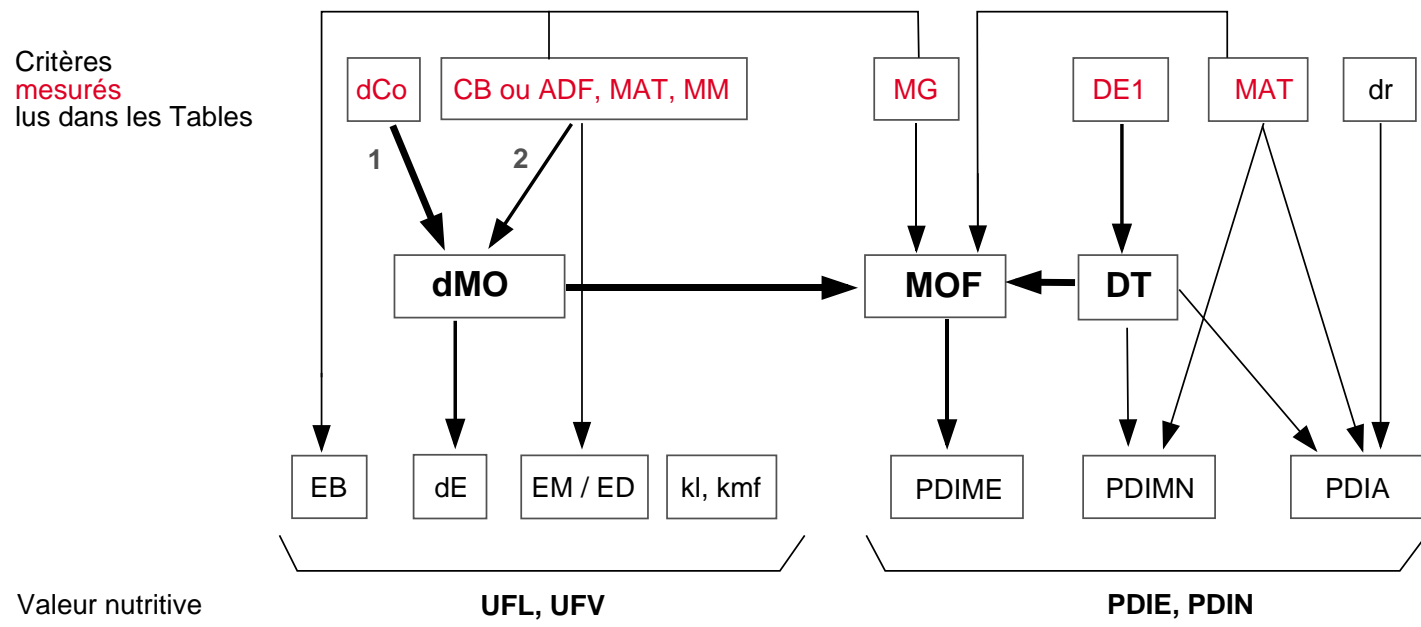

1, 2 : Ordre de priorité utilisé pour estimer la dMO

\section{Comme pour les fourrages, la valeur nutritive des concentrés est ba sée sur la dMO et la DT, PrévAlim utilisant en priorité les valeurs issues des mesures enzymatiques.}

équations proposées par Sauvant et al (1987). A la différence des fourrages, la teneur en matières grasses des aliments concentrés peut être saisie.

La nouvelle version d'INRAtion avec PrévAlim intègre les mesures les plus récentes réalisées sur les luzernes déshydratées (Demarquilly 1993 et Demarquilly et al 1996) et donne des valeurs allant jusqu'à des teneurs en MAT de $25 \%$. Les luzernes déshydratées sont classées dans les aliments concentrés puisque les valeurs d'encombrement n'ont pas été définies pour ces aliments. Toutefois la prévision de leur valeur énergétique s'apparente à celle des fourrages. La dMO peut être estimée soit à partir de la dCs ou de la dCo (Aufrère et Graviou 1996) ou à défaut à partir des teneurs en CB et MAT (Demarquilly et al 1996). Le calcul des valeurs UFL et UFV est réalisé avec les équations proposées par Demarquilly et al (1996).

PrévAlim estime la dMO et la valeur énergétique des aliments composés à partir des équations proposées par Giger et al (1990). Deux équations ont été retenues pour la prévision de la dMO : la première utilise la $\mathrm{dCo}$ et

\section{2 / Valeur azotée}

Le calcul des valeurs PDI des aliments concentrés nécessite de connaître la teneur en MAT, la DT et la dr de l'aliment, ainsi que la prévision de la dMO pour calculer la MOF. La DT et les valeurs PDI des aliments concentrés peuvent être prévues à l'aide de la dégradabilité de l'aliment pendant une heure d'incubation dans une solution contenant une protéase (DE 1, Aufrère et al 1989). Le module PrévAlim permet de saisir le résultat de DE 1 et de l'utiliser pour estimer les valeurs PDI de l'aliment. Dans le cas des aliments concentrés simples appartenant à une des douze classes définies par Aufrère et al (1989), les valeurs PDI sont calculées à partir de la DT prévue, de la dr des Tables, de la teneur en MAT et de l'estimation de la MOF (figure 5, tableau 2). Pour les aliments simples n'appartenant pas à une de ces douze classes de matières premières, les valeurs PDI sont alors estimées directement à partir de la teneur en MAT de l'aliment et de sa teneur en MANDE 1 (MAT*(1-DE 1)), qui intègre le résultat de la dégradabilité enzymatique. La valeur PDIE peut être prévue de façon plus précise si la 
Figure 6. Prévision de la valeur nutritive des aliments concentrés composées.

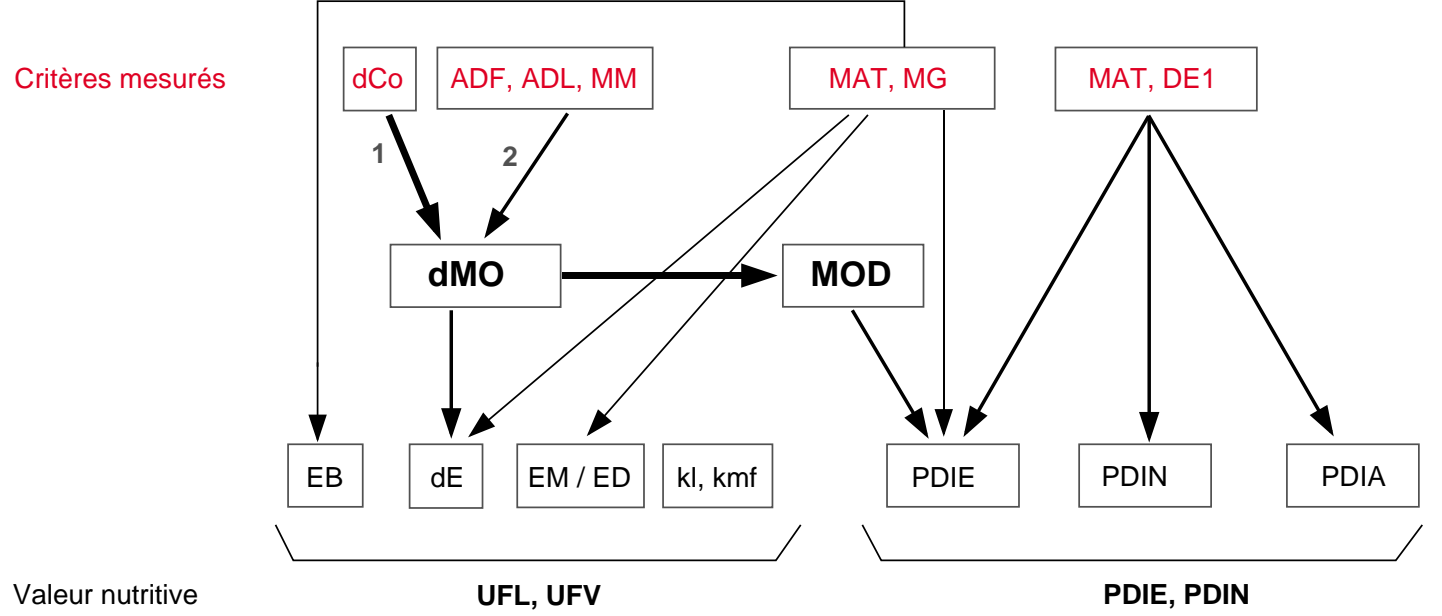

1, 2 : Ordre de priorité utilisé pour estimer la dMO

dMO et la teneur en matières grasses de l'aliment sont connues. PrévAlim permet aussi de saisir directement une valeur de DT ou bien de sélectionner celle des Tables, et de calculer alors la valeur PDI à partir de cette valeur et de la teneur en MAT de l'aliment.

Pour les aliments concentrés composés, la composition en matières premières n'est, le plus souvent, pas connue de l'utilisateur et la $\mathrm{dr}$ de l'aliment ne peut donc pas être calculée. La démarche consiste alors a prévoir directement les valeurs PDI à partir de la teneur en MAT et de la teneur en MANDE 1 selon les équations proposées par Aufrère et al (1989). Un gain de précision peut être obtenu sur la valeur PDIE si l'on peut estimer la dMO et si la teneur en matières grasses de l'aliment a été déterminée (figure 6 , tableau 2). Dans le cas des aliments composés contenant de l'urée, la connaissance du taux d'urée dans I'aliment est nécessaire. En effet l'urée n'apporte que des PDIN et le module de prévision corrige alors les teneurs en MAT et en MANDE 1 de la contribution de l'urée pour le calcul des valeurs PDIA et PDIE.

\section{4 / Structure informatique et fonctionnalités de PrévAlim}

PrévAlim est un module indépendant qui met à jour une base d'aliments de l'utilisateur à l'issue des calculs de prévision. L'interface entre PrévAlim et INRAtion est donc assurée automatiquement si PrévAlim est utilisé en amont d'INRAtion. Ecrit en Borland Turbo Pascal $7.0^{\circledR}$ comme INRAtion, ce module utilise trois sources de données:

- la base de référence INRA contenant 1287 aliments (dont 1156 fourrages et 131 concentrés) et 31 valeurs par aliment ;

- une base de paramètres pour les fourrages qui comprend 81 paramètres par fourrage :

- une base de paramètres pour les concentrés qui comprend 54 paramètres par concentré.

En pratique, sauf dans le cas des aliments concentrés composés, I'utilisateur doit d'abord sélectionner un aliment de référence (voir exemples dans le tableau 3) dans la Table INRAtion puisque certaines valeurs (MG, DT pour les fourrages, dr pour tous les aliments) y sont lues et que les équations utilisées pour la prévision de la dMO dépendent du type d'aliment. Le choix de cet aliment de référence doit donc être effectué de la façon la plus précise possible. Lorsque l'utilisateur ne dispose que d'une analyse incomplète, il peut saisir les valeurs disponibles et compléter par les valeurs de l'aliment de référence choisi. Par exemple, si pour un fourrage, la dCs et les MAT ont été analysées et que la CB n'a pas été analysée, le calcul peut être fait en sélectionnant la teneur en CB des Tables, celle-ci n'intervenant que dans le calcul du rapport EM/ED.

PrévAlim gère automatiquement les priorités entre les équations de calcul de la dMO, tout en laissant le choix à l'utilisateur de la méthode de calcul qui lui paraît la plus significative. Pour le calcul des autres valeurs (UF, PDI, UE), le programme utilisera les équations assurant la meilleure homogénéité avec l'équation retenue par l'utilisateur pour l'obtention de la dMO. Pour les fourrages, lorsque le module utilise une équation basée sur les teneurs en $C B$ et en MAT, il vérifie si les valeurs sont dans le domaine de validité de I'équation et si elles sont cohérentes entre elles. Dans le cas contraire les valeurs sont ramenées à la borne la plus proche. D'autres paramètres donnent les limites autorisées (âge de la plante par exemple) pour l'entrée des donnees. Enfin, certains paramètres d'équations de transformation permettent indifféremment la saisie d'une valeur sous deux formes: CB et ADF par exemple.

PrévAlim fonctionne en mode " tableur ". Le calcul des valeurs est effectué automatiquement dès lors que le programme dispose de suffisamment de données en entrée. Ainsi, la saisie des valeurs MAT et CB pour les fourrages et des valeurs de MAT, CB, MG et DT pour les concentrés suffit, dans la plupart des cas, à obtenir l'ensemble des valeurs (UF, PDI, UE ). En l'état, l'intégration ou l'interface 
de PrévAlim avec d'autres logiciels ne poserait pas de réel problème, dès lors que ces logiciels sauraient fournir à PrévAlim I'aliment de référence qui lui est nécessaire pour la récupération des paramètres des équations de calcul des valeurs.

\section{Conclusions et perspectives}

Le premier objectif de l'élaboration de PrévAlim était de faire la synthèse des outils existants pour prévoir la valeur des aliments pour les ruminants et de les organiser dans un outil informatique d'utilisation aussi simple que possible. PrévAlim rassemble, coordonne et met à jour les outils déjà disponibles pour estimer la valeur nutritive des aliments (Tables de prévision de 1981 et équations publiées depuis) et propose une approche rénovée pour estimer les valeurs UE. Les valeurs calculées (UF, PDI et UE) sont cohérentes entre elles ainsi qu'avec celles des Tables. L'approche hiérarchisée adoptée permet de calculer les valeurs même avec des informations manquantes et de retenir la méthode d'évaluation la plus pertinente en cas d'informations redondantes.

Cet outil est certainement perfectible. II bénéficiera des remarques des utilisateurs et facilitera le dialogue sur l'utilisation de méthodes de prévision de la valeur des aliments. L'individualisation des paramètres par aliment permettra une amélioration des estimations dès lors que seront disponibles de nouvelles valeurs et/ou de nouvelles équations. Comme pour INRAtion (Coulon et Agabriel 1992), cet outil pourra intégrer des révisions des Tables de la valeur des aliments, des améliorations des équations de prévision à partir des méthodes existantes ou de nouvelles méthodes, en particulier pour la valeur azotée des fourrages et l'ingestibilité des aliments (Baumont et al 1996).

La synthèse des différentes méthodes utilisées en France pour prévoir la valeur des aliments pour les ruminants met bien en évidence le rôle central de la prévision de la dMO. La dMO est le facteur de variation essentiel de la valeur énergétique et détermine fortement la valeur PDIE ainsi que l'ingestibilité des aliments. La possibilité de saisir directement dans PrévAlim une valeur de dMO permet d'utiliser des valeurs soit réellement mesurées, soit estimées par d'autres méthodes comme la digestibilite in vi tro avec du jus de rumen, différentes méthodes enzymatiques ou encore la spectrométrie dans le proche infra-rouge.

Le Iogiciel INRAtion (version 2.7) avec le module PrévAlim est diffusé par Educagri éditions, 26 bd Docteur Petitjean, 21036 Dijon Cedex. Contact : Philippe Beguyot, ENESAD-CNERTA, Tél : 03807727 18, e-mail : p. beguyot@educagri.fr

\section{Références}

Andrieu J., 1985. Composition et valeur alimentaire du maïs plante entière. In : Colloque Maiïs Ensilage, Rennes 29-30 Mai 1985 (AGPM ed).

Andrieu J., Aufrère J., 1996. Prévision à partir de différentes méthodes (physique, chimique et biologique) de la digestibilité et de la valeur énergétique de la plante de maïs à l'état frais. In : Colloque Maïs Ensilage, Nantes 1718 Septembre 1996. (AGPM ed), 61-69.

Andrieu J., Demarquilly C., 1987. Valeur nutritive des fourrages : tables et prévision. Bull. Tech. CRZV Theix, INRA, 70, 61-74.

Andrieu J., Demarquilly C., Sauvant D., 1988. Tables de la valeur des aliments. Les fourrages. In : R. J arrige (ed), Alimentation des Bovins, Ovins et Caprins, 356-444. INRA, Paris.

Andrieu J .P., Demarquilly C., Rouel J ., 1992. Conservation et utilisation par les génisses de I'herbe de prairies naturelles. Intérêt des balles rondes enrubannées comparativement à l'ensilage direct et au foin. INRA Prod. Anim., 5, 205-212.

Andrieu J ., Rouel J ., Perry Ch., Bony J ., 1997. Influence de l'hybride sur l'ingestibilité chez la vache laitière des ensilages de maïs plantes entières. Renc. Rech. Ruminants, 4 , 98.

Aufrère J., Demarquilly C., 1989. Predicting organic matter digestibility of forage by two pepsin-cellulase methods. In : XVI International Grassland Congress, Nice, France, 887-889.
Aufrère J., Graviou D., 1996. Prévision de la digestibilité des fourrages et aliments concentrés et composés des herbivores par une méthode enzymatique pepsine-celluIase. Note au BIPEA.

Aufrère J., Michalet-Doreau B., 1983. In vivo digestibility and prediction of digestibility of some by-products. In : Feeding values of by-products and their use by beef cattle, EEC Seminar, 26-29 September 1983. Melle Gontrode, (Belgique).

Aufrère J., Michalet-Doreau B., 1988. Comparison of methods for predicting digestibility of feeds. Anim. Feed. Sci., 20, 203-218.

Aufrère J., Graviou D., Demarquilly C., Vérité R., Michalet-Doreau B., Chapoutot P., 1989. Aliments concentrés pour ruminants : prévision de la valeur azotée PDI à partir d'une méthode enzymatique standardisée. INRA Prod. Anim., 2, 249-254.

Baumont R., Barlet A., Jamot J., 1996. L'effet d'encombrement ruminal des fourrages : sa relation avec l'ingestibilité et étude de sa prévision au laboratoire. Renc. Rech. Ruminants, 3, 313-316.

Baumont R., Dulphy J.P., Demarquilly C., 1997. Maximiser l'ingestion des fourrages conservés. Renc. Rech. Ruminants, 4, 57-64

Coulon I.B., Agabriel J ., 1992. Réflexions sur les outils de mise en œuvre des recommandations alimentaires. Application aux vaches laitières dans les conditions françaises. INRA Prod. Anim., 5, 347-353. 
Demarquilly C., 1993. Valeur énergétique des luzernes déshydratées. INRA Prod. Anim., 6, 137-138.

Demarquilly C., Andrieu J., 1988. Les fourrages. In : R. Jarrige (ed), Alimentation des Bovins, Ovins et Caprins, 315-336. INRA, Paris.

Demarquilly C., Andrieu J., Aufrère J ., Graviou D., 1996. Valeur énergétiques des luzernes. In : Attualita e prospettive della foraggicoltura da proto et da parcolo, Lodi (Italie), 22-24 Maggio.

Demarquilly C., Dulphy J.P., Andrieu J.P., 1999. Valeurs nutritive et alimentaire des fourrages selon les techniques de conservation : foin, ensilage, enrubannage. Fourrages, 155, 349-369.

Dubin L.M., Agabriel J ., Baumont B., Baumont R., Bolot C., Bourachot J.E., Coulon J.B., Champciaux P., J euland H., Riols P., 1998. Rationnement des vaches laitières : collaboration innovante entre pratique de terrain et recherche scientifique par l'élaboration d'un logiciel. Renc. Rech. Ruminants, 5, 260.

Dulphy J.P., Demarquilly C., Henry M. 1975. Pertes en composés volatils lors de la détermination de la teneur en matière sèche des ensilages. Ann. Zootech., 24, 743756.

Dulphy J.P., Faverdin Ph., Micol D., Bocquier F., 1987. Révision du système des unités d'encombrement. Bull. Tech. CRZV Theix, INRA, 70, 35-48.

Faverdin P., Delaby L., Vérité R., Marquis B., 1998. Effet de la teneur en protéines et en aliments concentrés d'une ration complète à base d'ensilage de maïs sur l'ingestion et la production laitière de vaches laitières en début de lactation. Renc. Rech. Ruminants, 5, 263.
Giger-Reverdin S., Aufrère J ., Sauvant D., Demarquilly C., Vermorel M., Pochet S., 1990. Prévision de la valeur énergétique des aliments composés pour les ruminants. INRA Prod. Anim., 3, 181-188.

Giovanni R., Scéhovic J., Peyraud J .L., Aufrère J ., 1992 Prévision de la digestibilité des graminées, des trèfles et des associations graminées-trèfle blanc à partir de leur composition chimique et de la digestibilité par la cellulase. Ann. Zootech., 41, 17-18.

INRA 1981. Prévision de la valeur nutritive des aliments des ruminants. (C. Demarquilly ed.). INRA, Paris, 580 p.

Rulquin H., Guinard J ., Vérité R., 1998. Variation of amino acid content in cattle small intestine digesta: development of a prediction model. Livest. Prod. Sci., 53, 1-13.

Sauvant D., Aufrère J. Michalet-Doreau B., Giger S. Chapoutot P., 1987. Valeur nutritive des aliments concentrés simples : tables et prévision. Bull. Tech. CRZV Theix INRA, 70, 75-90.

Valentin S.F., Forbes J. M., Lescoat P., 1999. Comparison of the voluntary intake by lactating cows of two maize silages with different in situ dry matter degradability. Ann. Zootech., 48, 211-218.

Vermorel M., Coulon J.B., J ournet M., 1987. Révision du système des Unités F ourragères. Bull. Tech. CRZV Theix, INRA, 70, 9-18.

Vérité R., Michalet-Doreau B., Chapoutot P., Peyraud J .L. Poncet C., 1987. Révision du système des protéines digestibles dans l'intestin. Bull. Tech. CRZV Theix, INRA, 70 19-34.

Abstract

\begin{abstract}
An integrated tool to predict feed value for ruminants: PrévAlim for INRAtion

This paper presents the bases of an integrated tool developed to estimate the feed value of forages and concentrates from their laboratory analysis. The computer program PrévAlim, available with the new version of INRAtion software, calculates the nutritive value of feed (feed unit UF and protein value PDI) and the fill unit (UE) of forages from the measurement of some characteristics of each feed. To calculate the nutritive value, a sequential approach is used, based on estimates of organic matter digestibility ( $\mathrm{dMO}$ ) and rumen degradability of feed protein (DT). UF and PDI values are then calculated using stepwise equations of the INRA (1988) systems. E stimates of the dMO are preferentially achieved from the enzymatic degradability method using pepsin-cellulase ( $\mathrm{dCs}$ or $\mathrm{dCo}$ ), or determined using the chemical composition of feed or the age of the plant. Estimates of the DT and the PDI values of concentrate feeds are achieved from the enzymatic degradability method using a protease (DE 1).
\end{abstract}

Fill values of forages are calculated from the estimates of their voluntary intakes based on their chemical com. position or dMO. Equations for different types of forages are calculated from the INRA (1988) feed tables. PrévAlim integrates, co-ordinates and updates different tools already available (prediction of nutritive value) and a new one that estimates fill values of forages. The computer program ensures that the calculated values (UF, PDI and UE) are coherent with themselves and with the feed tables. The hierarchical approach we used allows 1- to estimate feed value even in case of some missing information 2- to choose the most relevant estimates in case of redundant informa. tion.

BAUMONT R., CHAMPCIAUX P., AGABRIEL J ., ANDRIEU J., AUFRĖRE J., MICHALET-DOREAU B., DEMARQUILLY C., 1999. Une démarche intégrée pour prévoir la valeur des aliments pour les ruminants: PrévAlim pour INRAtion. INRA Prod Anim., 12, 183-194. 